\title{
Mechano-growth factor accelerates the proliferation and osteogenic differentiation of rabbit mesenchymal stem cells through the PI3K/ AKT pathway
}

Yanxiang Tong ${ }^{1}$, Wei Feng ${ }^{2}$, Yimin $W_{u^{2}}$, Huicheng $L v^{2}$, Yanfei Jia ${ }^{2}$ and Dianming Jiang ${ }^{{ }^{*}}$

\begin{abstract}
Background: Mesenchymal stem cells (MSCs) can differentiate into chondroblasts, adipocytes, or osteoblasts under appropriate stimulation. Mechano-growth factor (MGF) reportedly displays a neuroprotective effect in cerebral regions that were exposed to ischemia and is expressed in stromal cells of the eutopic endometrium and in glandular cells of the ectopic endometrium.

Results: This study sought to understand the potential involvement of phosphatidylinositol-3-kinase (PI3K)/protein kinase B (AKT) in MGF-induced growth of rabbit MSCs (rMSCs). We applied various concentrations of MGF to cultured rMSCs and observed the growth rate of the cells, the changes in the phosphorylation state of AKT and mammalian target of rapamycin (mTOR), and the expression levels of alkaline phosphatase and osteocalcin. We found that the growth and osteogenic differentiation of MGF-induced rMSCs were promoted primarily by phosphorylated AKT, and that this phosphorylation, as well mTOR phosphorylation, was mediated by the MGF receptor.
\end{abstract}

Conclusion: Our study suggests that MGF promotes the growth and osteogenic differentiation of rMSCs primarily through the PI3K/AKT pathway.

Keywords: rMSCS, PI3K/AKT, MGF, Osteogenic differentiation

\section{Background}

Mesenchymal stem cells (MSCs) can differentiate into chondroblasts, adipocytes, or osteoblasts under appropriate stimulation [1]. Adult rabbit MSCs (rMSCs) are an important source for tissue repair and therapy in regenerative medicine [2]. Therefore, expanding the osteogenic capacity of rMSCs is of major interest for improving the osteogenic potential of rMSCs for optimal bone regeneration [3]. The osteogenic differentiation of MSCs is characterized by the appearance of timely expressed genes, such as runt-related transcription factor 2, alkaline phosphatase (ALP) and type I collagen, followed by extracellular matrix mineralization [4].

\footnotetext{
* Correspondence: jiangdianmingtyx@163.com

'Department of Orthopedic, The First Affiliated Hospital of Chongqing Medical University, Chongqing, China

Full list of author information is available at the end of the article
}

Phosphatidylinositol-3-kinase (PI3K)/protein kinase B (AKT) signaling pathways are activated by a variety of extracellular stimuli and regulate a wide range of cellular processes, including cell motility, survival, proliferation, and cell cycle progression. Recent studies showed that the activation of PI3K/AKT is involved in cell survival and axonal outgrowth in neurons [5-8]. Mechanogrowth factor (MGF) has been found in many tissues. It reportedly displays a neuroprotective effect in cerebral regions that were exposed to ischemia and is expressed in stromal cells of the eutopic endometrium and in glandular cells of the ectopic endometrium. In recent years, increasing attention has been paid to MGF because of its regenerative effects on neurons, human mesenchymal stem cells, and osteoblasts $[9,10]$. In addition, tissue-protective actions of MGF have been 
demonstrated in acute myocardial infarction, neuron damage, and rabbit bone-defect models [11].

Here, we used MGF to investigate the molecular mechanisms underlying MSC osteoblast differentiation and enhance the osteogenic potential of rabbit MSCs. Our data revealed that MGF through PI3K/AKT signaling pathways trigger osteogenic differentiation of rabbit bone marrowderived mesenchymal stromal cells.

\section{Results}

\section{Identification of rMSCs}

The morphology of the rMSCs was determined using a microscope (Figure 1A). At day 10, cells reached $80 \%$ confluence. At day 13, the cells displayed a uniform spindle shape and reached $100 \%$ confluence. To further identify the rMSCs, CD34 and CD44 cell markers were examined. Immunofluorescence staining indicated that the CD44 cell marker fluoresced green on the cell surface under a fluorescent microscope, whereas CD34 fluoresced brown (not green), indicating that cultured cells were positive for CD44, but negative for CD34 (Figure 1B).

The proliferation of the cells for the second through the fifth cell passages was analyzed with an automated microplate reader at an absorbance of $570 \mathrm{~nm}$ on days 1-10. As shown in Figure 1C, the growth curves for the passaged cells displayed " $S$ " shapes, with the latent growth phase of the passaged cells stabilizing during the first to second days. The cells from the fourth and fifth passages were $100 \%$ confluent at 7 days and then entered a lag phase. However, the cells from the second and third passages entered the lag phase 2 days later. Thus, the cells from the fourth passage displayed the greatest ability to proliferate $(\mathrm{P}<0.05)$.

\section{MGF receptor expression in $\mathrm{rMSCs}$}

To detect the expression of MGF receptor in rMSCs, we used immunocytochemistry techniques, and Figure 2 shows MGF receptors expressed in rMSCs. MGF expressed in the cytoplasm.

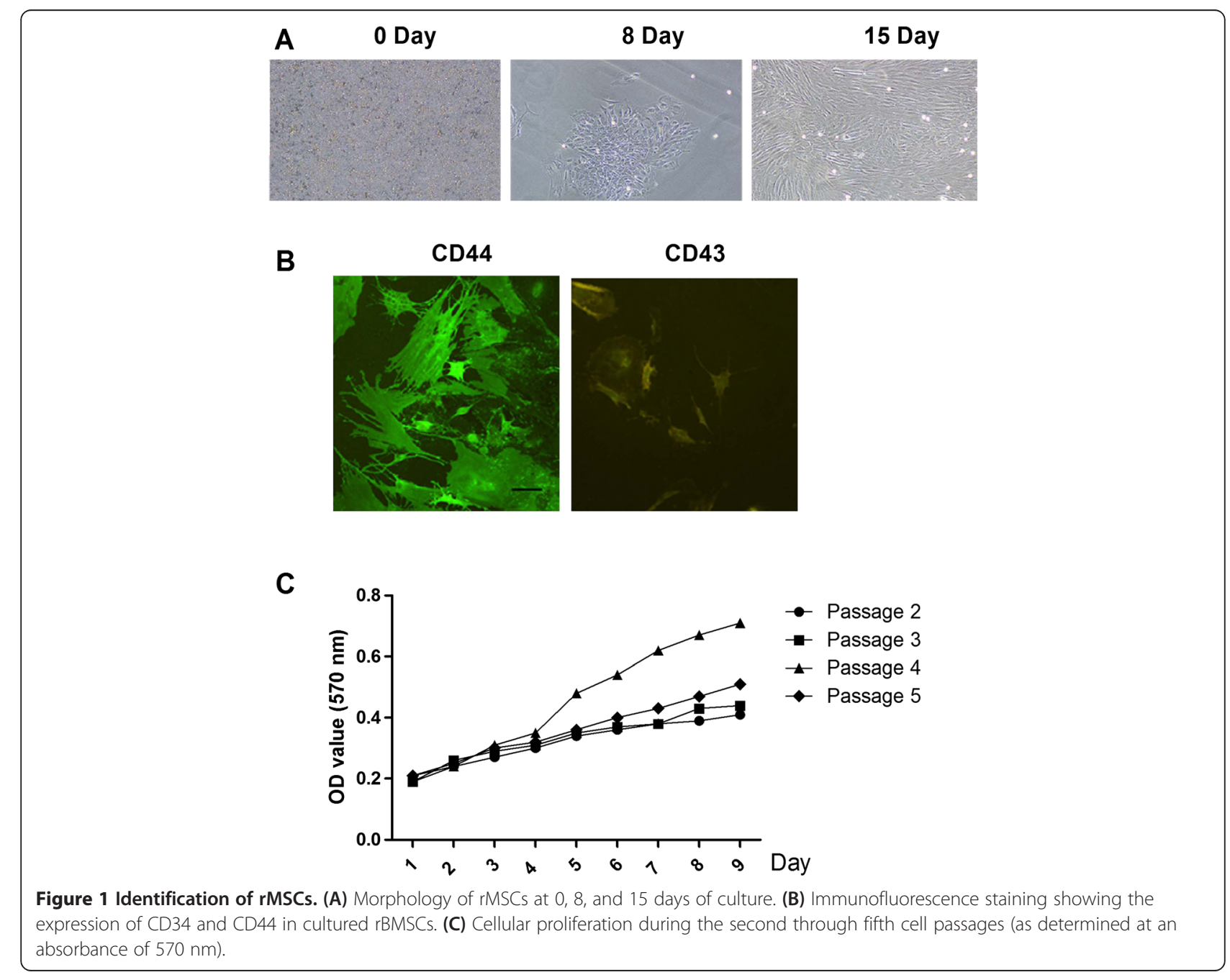




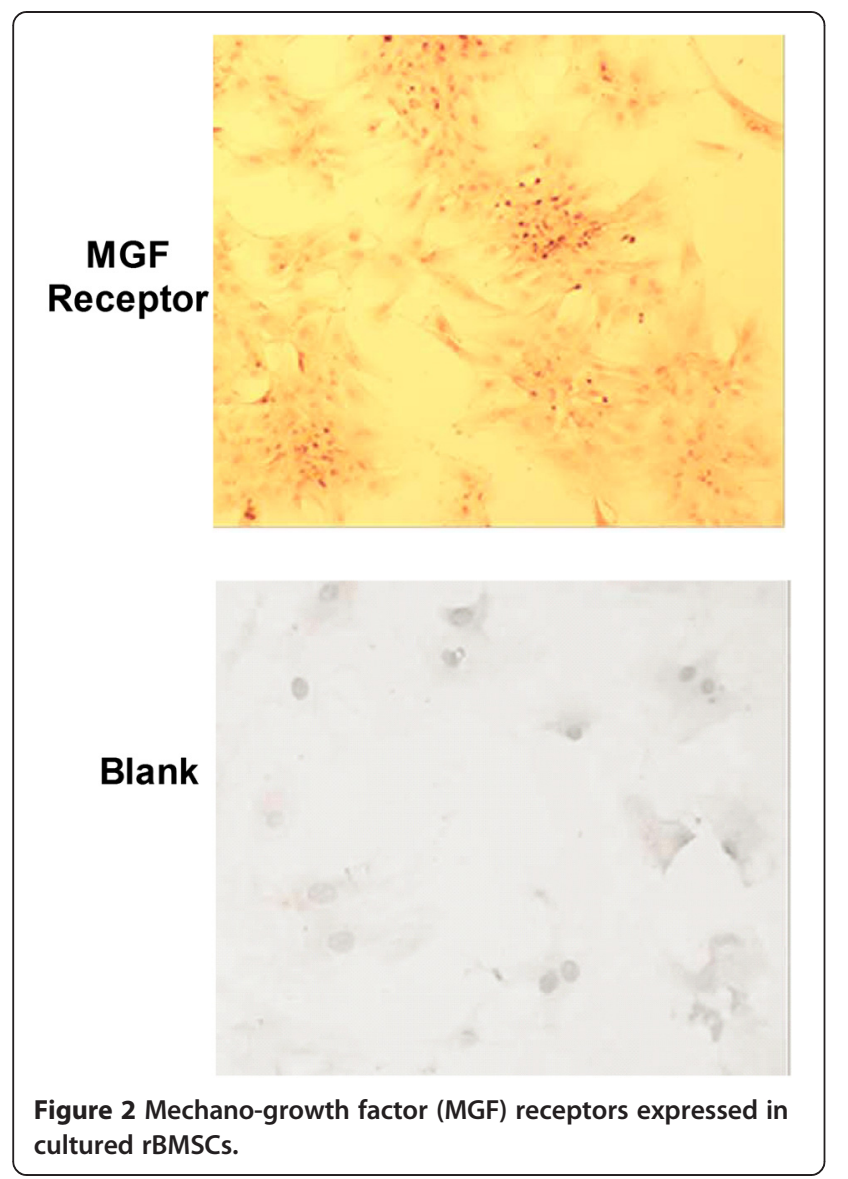

Optimal MGF concentration and application time for MGF-induced effects on rMSCs

The optimal MGF concentration and application time for the MGF-induced effects on the rMSCs were determined. Figure 3 shows the growth rates of rMSCs from the fourth cell passage for 1-10 days following the addition to the cell culture media of $15,30,45,60$, or $75 \mathrm{ng} / \mathrm{ml}$ of MGF. The maximum growth rate appeared at an MGF concentration of $45 \mathrm{nM}$ between days 4 and 5 .
MGF promotes the growth and differentiation of rMSCs via the PI3K/AKT pathway

To identify the signaling pathway through which MGF acts on rMSCs, cells were treated with $45 \mathrm{nM}$ of MGF for 5 days (the optimal conditions as determined above), and the phosphorylation states of AKT and mTOR were examined at $0,4,8,12$, and $16 \mathrm{~h}$ following treatment on day 5 (Figure 4A). Figure 4B shows that the phosphorylation of AKT and mTOR increased. To further explore the function of MGF, the expression levels of ALP and OCN were examined in the osteoblasts that were formed from the rMSCs. The expression of these two proteins was higher at $4 \mathrm{~h}$ that at other time in cells treated with $45 \mathrm{nM}$ MGF for $4 \mathrm{~h}$ on the fifth day of treatment (Figure 5).

To verify that MGF promotes the growth and differentiation of rMSCs via the PI3K/AKT pathway, the PI3K/ AKT inhibitor LY294002 with $20 \mu \mathrm{mol} / \mathrm{L}$ was used to inhibit PI3K/AKT activity (Figure 6A). Following the addition of LY294002, the cells were treated with MGF for 1-10 days, and their growth rates were compared with those of cells that did not receive LY294002 treatment (Figure 6B). We found that the phosphorylation of mTOR was decreased compared with that of controls (Figure 6A). The inhibition of PI3K/AKT also blocked the ability of MGF at $45 \mathrm{nM}$ to increase the growth rate of rMSCs, even after 10 days of treatment. In addition, the expression levels of ALP and OCN were decreased compared with those in control osteoblasts (Figure 7).

\section{Discussion}

Finding mechanisms that trigger the growth and osteogenic differentiation of rMSCs may help develop novel therapeutic approaches to promote bone formation [12]. In the present study, we demonstrated that MGF promoted the growth rate and osteogenic differentiation of rMSCs via the PI3K/AKT pathway.

The identity of rMSCs were verified by their morphology and the finding that the rMSCs expressed CD44, a marker of rMSCs, but not CD34, a closely

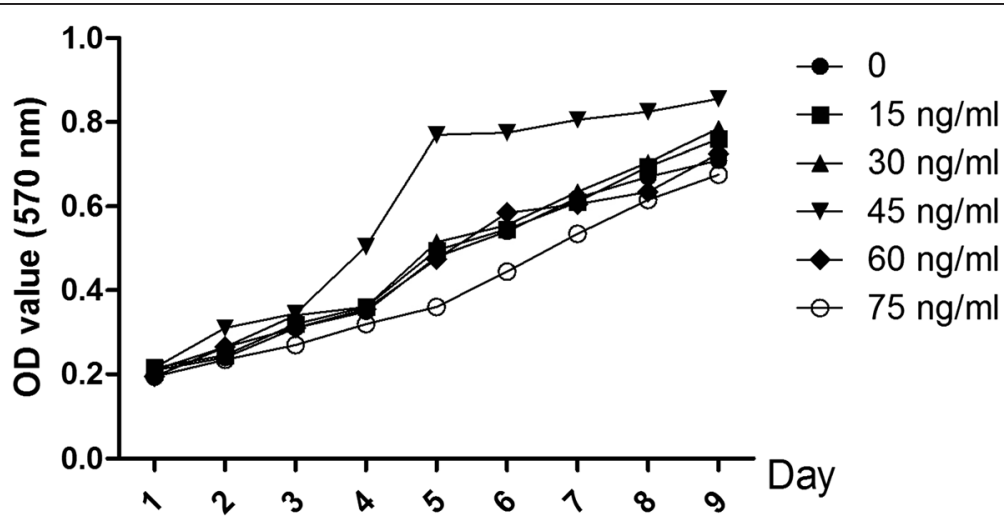

Figure 3 Growth rates of cultured rMSCs treated for 1-10 days with MGF (15, 30, 45, 60, or $75 \mathrm{ng} / \mathrm{ml})$. 


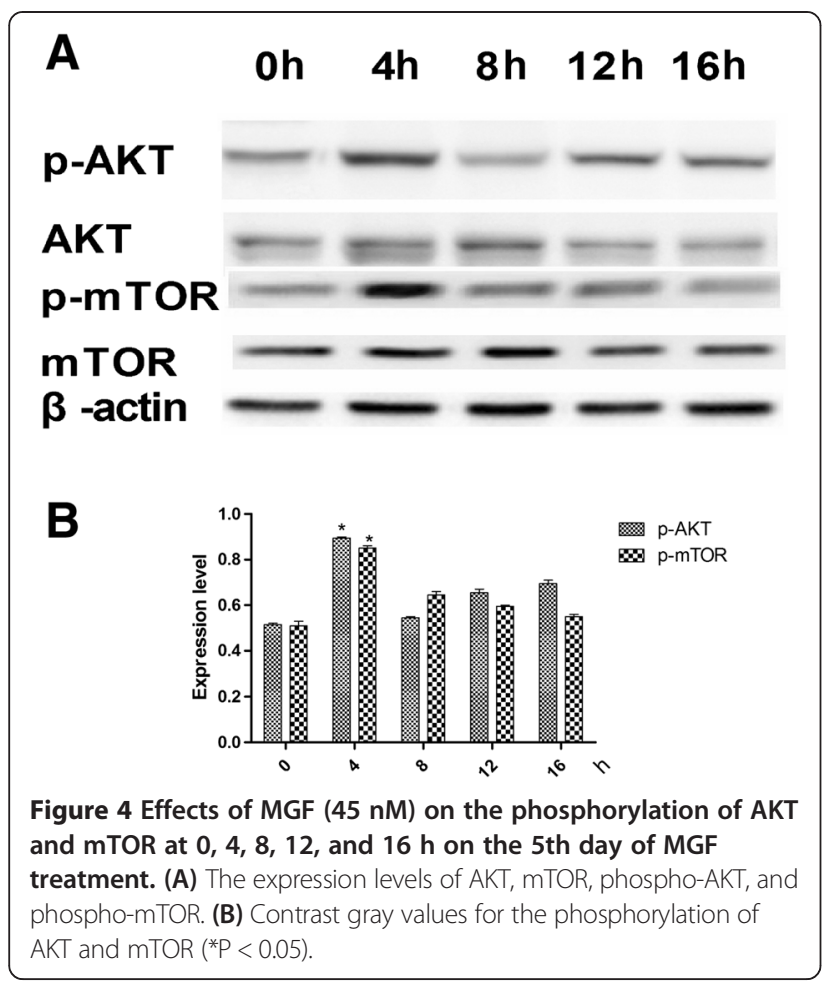

related molecule that does not label rMSCs. Cellular proliferation following the second through fifth passages was analyzed on days 1-10 following the passages. The fourth generation rMSCs showed the greatest capacity for proliferation, whereas the second generation showed the weakest.

We determined that the maximum growth rate of rMSCs was achieved by the addition of $45 \mathrm{nM}$ MGF to the culture medium for 5 days. We also demonstrated that the MGF receptor is expressed at high levels in rMSCs.

To gain further insight into the mechanisms through which MGF promotes growth and differentiation in rMSCs, we evaluated the activity of the signaling pathways downstream of the MGF receptor. The PI3K/ AKT/mTOR signaling pathway plays a crucial role in

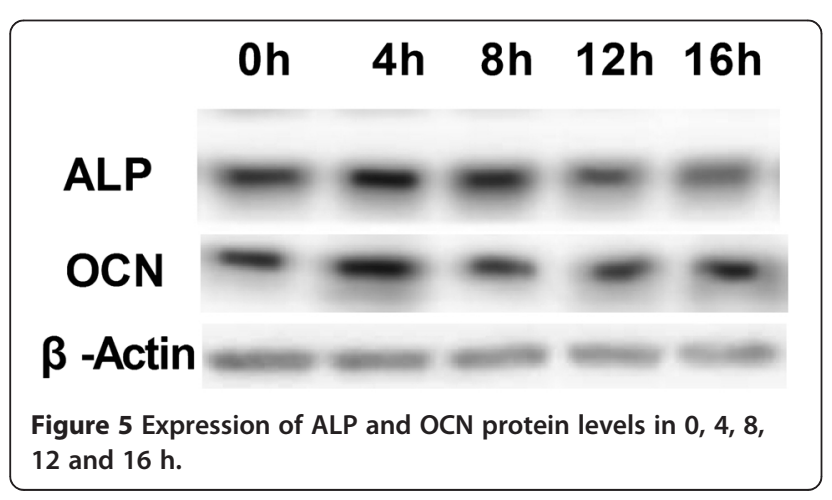

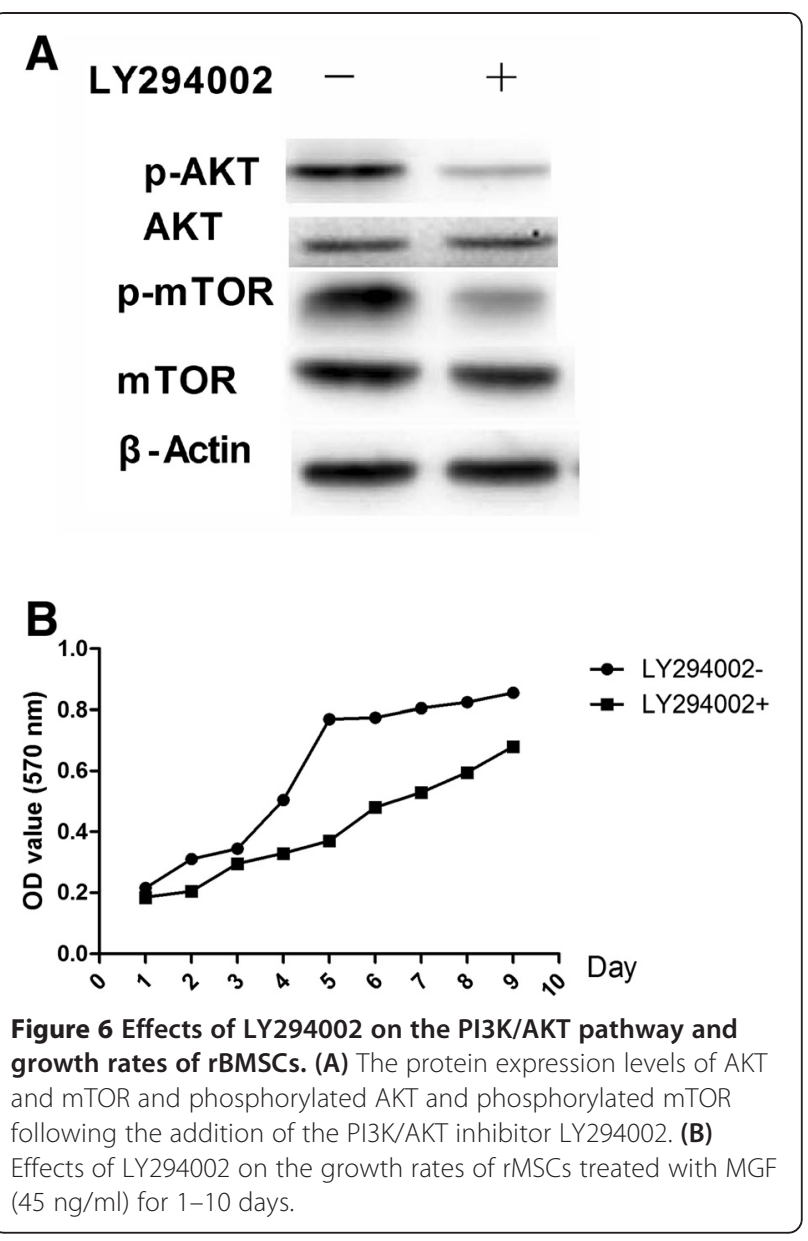

LY294002 - +

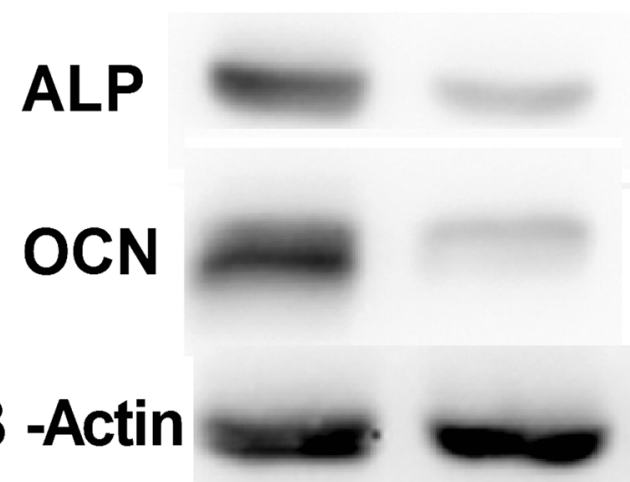

Figure 7 Effects of LY294002 on the expression of ALP and OCN protein levels. 
multiple cellular processes, including cell proliferation, angiogenesis, metabolism, differentiation, and survival [13]. The results of our present study suggested that MGF promoted the growth of rMSCs via the PI3K/AKT signaling pathway. We found that $4 \mathrm{~h}$ on the fifth day of MGF treatment was required to stimulate maximal phosphorylation of AKT and mTOR and maximal expression of ALP and OCN. Furthermore, inhibiting AKT with LY294002 suppressed the MGF-mediated promotion of growth and osteogenic differentiation in rMSCs.

\section{Conclusion}

Overall, our study suggests that the MGF receptor signaling pathway accelerates growth in rMSCs primarily through a PI3K/AKT-dependent pathway. Despite this promising finding, further study is necessary before any clinical applications can be considered.

\section{Methods}

Unless otherwise specified, all chemicals and reagents were purchased from the Sigma Chemical Company (St. Louis, MO, USA). MGF was purchased from Chemicon International (Temecula, CA, USA). Antibodies to IgG, $\beta$-actin, AKT, mammalian target of rapamycin (mTOR), ALP, osteocalcin (OCN), CD44, CD34, MGF receptor, phospho-AKT, phospho-mTOR, and phospho-p90RSK1 (Ser380) were purchased from the Millipore Corporation, USA.

\section{Isolation and culture of rMSCs}

The femur from a neonatal New Zealand white rabbit was isolated, and the ends of the femur were opened. The bone marrow was flushed from the femur with low glucose Dulbecco's modified Eagle's medium (DMEM) using a $1 \mathrm{~mL}$ syringe. Cells were harvested in a culture dish, dissolved using a Pasteur pipette, seeded onto a flask containing DMEM and 15\% fetal bovine serum, and cultured in an incubator with $5 \% \mathrm{CO} 2$ at $37^{\circ} \mathrm{C}$. The medium was replaced every 2 days. When cells grew to a confluence of approximately $85 \%$, they were passaged with $0.25 \%$ trypsin and $0.1 \%$ EDTA (1:2). Cell growth was monitored under an inverted phase contrast microscope (Nikon Co.). The animal test was agreed by The Chongqing Medical University Experimental Animal Management Committee.

\section{3-(4,5-Dimethylthiazol-2-yl)-2,5-diphenyltertrazolium bromide (MTT) assay}

Cells were grown in 96-well plates $\left(1 \times 10^{3}\right.$ cells/well $)$ supplement with MGF. Control cells were switched from RPMI-1640 to DMEM containing 0.1\% dimethyl sulfoxide (DMSO). At 1, 2,3, 45,6,7,8 and 9 days following MGF treatment $(0,15,30,45,60$ and $75 \mathrm{nM}$ MGF), $20 \mu \mathrm{L}$ of MTT was added to each well to a final concentration of $0.5 \%$. After a $4 \mathrm{~h}$ incubation at $37^{\circ} \mathrm{C}$ in the dark, $150 \mu \mathrm{L}$ DMSO was added to each well for $10 \mathrm{~min}$ to dissolve the formazan crystals. The absorbance was measured using a microplate reader (EXL800, Cole-Parmer, Vernon Hills, IL, USA) at $490 \mathrm{~nm}$. All experiments were repeated three times. The viability of the MGF treated cells was expressed as percentage of population growth plus the standard error of the mean (SEM) relative to that of untransfected control cells. Cell growth was calculated as follows: \% growth $=($ mean experimental absorbance- mean control absorbance/mean control absorbance) $\times 100$.

\section{Immunofluorescence}

The rMSCs were fixed in $3.7 \%$ paraformaldehyde for $30 \mathrm{~min}$ at room temperature, permeabilized with $0.5 \%$ Triton X-100 in PBS for 15 min, and blocked with 1\% BSA in phosphate buffered saline (PBS) with $10 \%$ goat serum overnight at $4^{\circ} \mathrm{C}$. The samples were then stained with primary antibodies diluted in PBS. The primary antibody binding was detected with an Alexa Fluor 488 Goat anti-rabbit IgG $(\mathrm{H}+\mathrm{L})$ secondary antibody. Images were captured with a Nikon A1 confocal microscope. Experiments were performed in triplicate.

\section{Immunohistochemistry}

Immunohistochemistry was performed as follows: cell sections were three times for $1 \mathrm{~min}$ in phosphate buffered saline (PBS), once fixed for $15 \mathrm{~min}$ in ice acetone, three times for 2 min in PBS, 0.5\% Triton X-100 20 min. Antigens were recovered by heating the sections in a microwave oven for $15 \mathrm{~min}$, after which the sections were washed three times for $5 \mathrm{~min}$ with PBS. Endogenous peroxidase activity was blocked by soaking the slides in a solution of $3 \%$ hydrogen peroxide for $15 \mathrm{~min}$ at room temperature (RT), followed by washing three times for $5 \mathrm{~min}$ with PBS. Non-immune Goat blood serum $(50 \mu \mathrm{L})$ was added to each section for $15 \mathrm{~min}$ at $\mathrm{RT}$, followed by the primary antibodies (Millipore, $50 \mu \mathrm{L}$ ). Slides were incubated overnight at $4^{\circ} \mathrm{C}$ in a humidified chamber and washed three times for $10 \mathrm{~min}$ in PBS. Biotin-labeled secondary antibodies $(50 \mu \mathrm{L})$ were added to each section and kept for $15 \mathrm{~min}$ at $37^{\circ} \mathrm{C}$, followed by washing three times for $5 \mathrm{~min}$ with PBS. Samples were incubated for $15 \mathrm{~min}$ at RT and washed three times for $5 \mathrm{~min}$ in PBS before the addition of $100 \mathrm{~L}$ freshly prepared 3,3-diaminobenzidine (DAB) for approximately 5$20 \mathrm{~min}$. The reaction was stopped by washing in cold water. Slides were counterstained with hematoxylin, followed by a sealing procedure using neural gum.

\section{Western blot}

The protein homogenates from human neurons were separated using electrophoresis on $8-12 \%$ sodium dodecyl 
sulphate/polyacrylamide gels and transferred to immunoblot nitrocellulose membranes. Membranes were blocked for $30 \mathrm{~min}$ at room temperature in PBS buffer containing $5 \%$ fat-free milk and $0.1 \%$ Tween 20 . Membranes were then incubated with primary antibody for at least $1 \mathrm{~h}$ at room temperature or overnight at $4^{\circ} \mathrm{C}$. The membranes were subsequently washed three times with PBS containing $0.1 \%$ Tween 20 , incubated with peroxidase-conjugated secondary antibodies, and developed using ECL reagents (Pierce, Rockford, IL, USA).

\section{Osteogenic differentiation}

The rMSCs were plated at a density of 5000 cells $/ \mathrm{cm}^{2}$ and exposed to standard differentiation-inducing media for 21 days. The medium was changed twice per week. Osteogenic differentiation was achieved following standard in vitro protocols. Endothelial differentiation was stimulated by culturing the cells in Endothelial Growth Medium-2 (EGM-2).

\section{Statistical analysis}

Statistically significant differences between gene expression levels were determined using one-way analysis of variance (ANOVA) followed by a Newman-Keuls test with GraphPad Prism version 5 software (GraphPad Software, La Jolla, CA, USA, www.graphpad.com/company/). Replicates were included in the statistical model. Differences were considered statistically significant at the $95 \%$ confidence level $(\mathrm{P}<0.05)$. Data are presented as mean \pm S.D.

\section{Abbreviations}

MSCs: Mesenchymal stem cells; MGF: Mechano-growth factor; PI3K/ AKT: Phosphatidylinositol-3-kinase /protein kinase B; rMSCs: rabbit MSCs.

\section{Competing interests}

The authors declare that they have no competing interests.

\section{Authors' contributions}

The authors declare that they have no competing interests. Authors' contributions YT carried out experimental design, statistical analysis, manuscript and figures preparation. YW and WF carried out experimental design, Western blot experiments, figure preparations, $\mathrm{HL}$ and $\mathrm{YJ}$ carried out cell culture, DJ carried out experimental design. All authors read and approved the final manuscript.

\section{Acknowledgements}

This work was acknowledge to the Dianming Jiang.

\section{Author details}

'Department of Orthopedic, The First Affiliated Hospital of Chongqing Medical University, Chongqing, China. ${ }^{2}$ The Second Affiliated Hospital of Inner Mongolia Medical University, Hohhot, China.

Received: 10 December 2014 Accepted: 7 January 2015

Published online: 15 January 2015

\section{References}

1. Wei Z, Chen N, Guo H, Wang X, Xu F, Ren Q, et al. Bone marrow mesenchymal stem cells from leukemia patients inhibit growth and apoptosis in serum-deprived K562 cells. J Exp Clin Cancer Res. 2009;28:141.
2. Zhang W, Zhang F, Shi H, Tan R, Han S, Ye G, et al. Comparisons of rabbit bone marrow mesenchymal stem cell isolation and culture methods in vitro. PLoS One. 2014;9:e88794.

3. Lapi S, Nocchi F, Lamanna R, Passeri S, lorio M, Paolicchi A, et al. Different media and supplements modulate the clonogenic and expansion properties of rabbit bone marrow mesenchymal stem cells. BMC Res Notes. 2008;1:53.

4. Huang CY, Reuben PM, Cheung HS. Temporal expression patterns and corresponding protein inductions of early responsive genes in rabbit bone marrow-derived mesenchymal stem cells under cyclic compressive loading. Stem Cells. 2005;23:1113-21.

5. Wang W, Wen Q, Xu L, Xie G, Li J, Luo J, et al. Activation of Akt/mTOR pathway is associated with poor prognosis of nasopharyngeal carcinoma. PLoS One. 2014;9:e106098.

6. Kanteti R, Dhanasingh I, Kawada I, Lennon FE, Arif Q, Bueno R, et al. MET and PI3K/mTOR as a potential combinatorial therapeutic target in malignant pleural mesothelioma. PLoS One. 2014;9:e105919.

7. Correction. AKT inhibitors promote cell death in cervical cancer through disruption of mTOR signaling and glucose uptake. PLoS One. 2014:9:e107846.

8. Liang M, LV J, Chu H, Wang J, Chen X, Zhu X, et al. Vertical inhibition of $\mathrm{PISK} / \mathrm{Akt} / \mathrm{mTOR}$ signaling demonstrates in vitro and in vivo anti-fibrotic activity. J Dermatol Sci. 2014;76(2):104-11.

9. Xin C, Bingbing Z, Yuanliang W, Chengyu X, Li Y, Moyuan D, et al. Mechano-growth factor $E$ peptide inhibits the differentiation and mineralization of osteoblasts. Arch Oral Biol. 2012;57:720-7.

10. Song L, Zhu WS, Dong JL, Li ZY, Liu XH. Expression and purification of two alternative peptides for mechano-growth factor in Escherichia coli. Biotechnol Lett. 2012;34:231-7.

11. Zhang B, Luo Q, Mao X, Xu B, Yang L, Ju Y, et al. A synthetic mechanogrowth factor $E$ peptide promotes rat tenocyte migration by lessening cell stiffness and increasing F-actin formation via the FAK-ERK1/2 signaling pathway. Exp Cell Res. 2014;322:208-16.

12. Wu J, Wu K, Lin F, Luo Q, Yang L, Shi Y, et al. Mechano-growth factor induces migration of rat mesenchymal stem cells by altering its mechanical properties and activating ERK pathway. Biochem Biophys Res Commun. 2013;441:202-7.

13. Zhu L, Derijard B, Chakrabandhu K, Wang BS, Chen HZ, Hueber AO. Synergism of PI3K/Akt inhibition and Fas activation on colon cancer cell death. Cancer Lett. 2014;354:355-64.

\section{Submit your next manuscript to BioMed Central and take full advantage of:}

- Convenient online submission

- Thorough peer review

- No space constraints or color figure charges

- Immediate publication on acceptance

- Inclusion in PubMed, CAS, Scopus and Google Scholar

- Research which is freely available for redistribution 